\title{
Dynamics in non-binding procurement auctions with boundedly rational bidders.
}

\author{
Domenico Colucci, Nicola Doni and Vincenzo Valori * \\ Facoltà di Economia, Università di Firenze
}

June 18, 2009

\begin{abstract}
We study a procurement auction recently analysed by Gal-Or et al. (2007). In this auction game the buyer ranks different bids on the basis of both the prices submitted and the quality of each bidder that is her private information. We emphasise the similarity between this model and existing models of competition in horizontally differentiated markets. Finally we illustrate conditions for the existence and the stability of such equilibrium. To this end we extend the model to a dynamic setting in which a sequence of independent auctions takes place. We assume bidders have bounded rationality in a twofold sense. On one hand, they use an underparametrized model of their competitors' behaviour, best responding to expectations on average bids rather than keeping track of the entire vector of competitors' bids. On the other they update expectations adaptively. In a general framework with more than two bidders the system may fail to converge to the steady state, i.e. to the symmetric Nash equilibrium of the original game.

Journal of Economic Literature Classification Numbers: D43, D44, C62, D83

Key Words: Non-binding auctions, Product differentiation, Hotelling Duopoly, Expectations, Stability of steady states
\end{abstract}

\section{Introduction}

Auction theory has always recognised that in many settings bidders' strategies can be influenced by the revelation of some information that is privately held by the auctioneer. Usually it is assumed that the auctioneer holds some information regarding the item put up for auction. As a consequence, its revelation can allow bidders to have a more

${ }^{*}$ We wish to thank Prof. Domenico Menicucci and an anonymous referee for valuable comments and suggestions. The usual caveat applies. 
accurate estimate of their valuation for the object and to make less uncertain their utility in case their bid is accepted ${ }^{1}$.

Some recent papers investigate the importance of a different kind of auctioneer's private information: in multidimensional auctions, bidders can be ignorant about the real awarding rule. Katok et al. [9] define this competitive mechanism as "non-binding auctions". More specifically, it is often assumed that a buyer can rank different bids according not only to the prices, but also to the quality associated to each proposals. The qualitative assessment usually depends on buyer's preferences that can be her private information because they are related to her tastes or to her specific requirements 2 . In this case bidders can always calculate thoroughly the ex-post profit associated to each specific bid; however, the information policy adopted by the buyer influences their estimate of the probability to be the winner. When the buyer chooses to reveal privately (publicly) her information suppliers are involved in a standard auction setting, with independent private (public) values. Conversely, the case in which the buyer conceals her information represents a novelty in the auction literature, and that is why we want to explore in more depth the characteristics of this game and the properties of its Nash equilibrium.

In the next section we introduce the general model of an auction where the buyer conceals her private information, as proposed by Gal-Or et al. [7] and we show that this specific setting is closely related to classic models of horizontal differentiation. In particular, we emphasise how, in the case with only two bidders, their equilibrium bidding strategies are equivalent to duopolists' pricing strategies in the Nash equilibrium of an Hotelling model with exogenous location. For this purpose we follow the recent generalization of the Hotelling model put forward by Kim [10]. In the general case of $n$ bidders a multidimensional auction with concealment of buyer's private information is formally identical to the model of product differentiation studied by Perloff and Salop [12]: the only difference is the analysis of the strategic value of the buyer's private information in Gal-Or et al. [7] with respect to the otherwise more general model of Perloff and Salop [12].

In section 3 we study a simple dynamic version of the above model. To this end we posit a sequence of auctions take place in time, to which a given set of suppliers

\footnotetext{
${ }^{1}$ Milgrom et al. 11 represents the seminal paper on this issue. By analysing an affiliated values auction model they stated the celebrated linkage principle, according to which expected revenue increases if the auctioneer commits to reveal any information about the value of the object. More recently some authors have shown that this principle can be wrong in some different contexts. See Ganuza [8], Board [4].

${ }^{2}$ See Gal-Or et al [7], Rezende [15], Katok et al. [9] for an analysis of this issue in procurement settings. Cason et al. [5] and Chan et al. [6] emphasise how this issue affects the awarding of subsidies in natural resource management programs. Note that a secret award rule is often present also in the procedures for the privatization of previously State-owned enterprises: Governments usually compare different proposals and at the end they select one private firm on the basis not only of the economic offers, but also of different factors, like political, social and environmental considerations.
} 
participate without actually knowing the quality assessments held by the auctioneers. It is thus a situation in which a given set of suppliers compete repeatedly to procure a specific good. We simply assume that every buyer is characterized by a vector of quality assessments, one for each supplier ${ }^{3}$. From the suppliers standpoint, buyers' assessments correspond to independent random draws from a given probability distribution. Each supplier maintains some kind of expectation regarding their opponents' behaviour which we shall suppose to be wrapped up in an expectation about a mean of the opponents' prices. Clearly this hypothesis qualifies agents as having bounded rationality, in that the opponents are treated as if they were one, whereas a fully rational player would have to figure out the best response to the predicted bids of every other player. This depends on the fact that these auctions are non-binding, so that qualities as well as bids determine the winner. While considering a mean price is clearly suboptimal, it nonetheless has the property of depending on the entire set of choices by the competitors as implied by full rationality. On the contrary, concentrating only on the others' best price, as would be optimal in standard auctions, is not rational in this context because the contract is not necessarily awarded to the lowest bidder. In turn expectations about the opponents' mean price are updated adaptively. This model entails a moderate departure from rationality in the vicinity of the steady state, which turns out to be unique and implies coordination on the Nash equilibrium of the stage game, and our purpose is precisely to study local stability: these observations are the rationale for using the above limited rationality model of choice. Alternatively the dynamic model can be seen in the Hotelling framework as a straightforward way to model the repeated situation of market competition in a horizontal differentiated oligopoly with boundedly rational sellers. The vast literature on oligopoly dynamics focuses mainly on the Cournot model and is neatly surveyed by Barkley Rosser [3]. Relatively fewer papers examine the dynamics in the Hotelling setting (for an example, see Puu and Gardini [14]). However they focus on the spatial competition, in which firms choose both a price and a location. Conversely, in the present paper we analyse the dynamics of (price-only) competition in the framework formalized by Perloff and Salop [12]. The stability of the dynamical system is not easy to assess in general because the reaction functions may be nondifferentiable at the steady state. In such a case the analysis can resort to the derivative of the reaction function in a neighborhood of the steady state. We will show how the stability of the Nash equilibrium of this game is affected by the distribution from which bidders' qualities are drawn. More specifically if $n=2$ then the Nash equilibrium is always stable whatever the quality distribution. Conversely when $n>2$ stability can be violated. In fact, if bidders' qualities are drawn from a specific class of densities then in equilibrium reaction functions are negatively sloped and the system may fail to converge.

\footnotetext{
${ }^{3}$ We could also imagine the case in which there is a unique buyer who, perhaps due to frequent job rotation related to political evolution, has time-changing quality assessments.
} 


\section{Auctions with horizontally differentiated suppli- ers}

\subsection{Equilibrium in non-binding auctions}

Assume there is a unique buyer wishing to procure a single unit of a specific product by means of an auction procedure. There are $n$ firms, competing to supply the item. Both the buyer and the suppliers are assumed to be risk-neutral. We allow the buyer to value the specific product provided by each seller differently. Let $q_{i}$ denote the buyer's evaluation of the quality associated to the bid of supplier $i$. We assume that the quality parameters are independent and identically distributed (i.i.d.) random variables with a continuous density $f$ (with cumulative distribution function $F$ ) over the support $[\underline{q}, \bar{q}]$ and that their realisations are privately known by the buyer only.

The utility the buyer can obtain contracting with a specific supplier depends on the quality of his product and the price asked to provide it:

$$
U\left(q_{i}, p_{i}\right)=q_{i}-p_{i} \quad i=1, \ldots, n .
$$

A multidimensional auction is held in order to select a supplier, and we assume that the score function used to rank alternative bids is the same as the buyer's utility function ${ }^{4}$. Suppliers are characterized by identical production costs, normalized to be 0 . Every competing bidder submits an economic bid $p_{i}$ in order to maximise his expected profit, equal to his ex-post profit times the probability of being the selected contractor:

$$
\max _{p_{i}} p_{i} \operatorname{Pr}\left\{q_{i}-p_{i} \geq \max _{j \neq i} q_{j}-p_{j}\right\}
$$

By taking into account how the quality of each competitor is distributed we can rewrite the maximization problem as follows:

$$
\max _{p_{i}} p_{i} \int_{\underline{q}}^{\bar{q}}\left[\prod_{j \neq i} F\left(q_{i}+p_{j}-p_{i}\right)\right] f\left(q_{i}\right) d q_{i}
$$

Further, restricting the attention only to symmetric equilibria and assuming the common bid submitted by competitors other than $i$ equals $\bar{p}$ the above rewrites as

$$
\begin{aligned}
& \max _{p_{i}} H\left(p_{i}, \bar{p}\right) \\
& H\left(p_{i}, \bar{p}\right) \equiv p_{i} \int_{\underline{q}}^{\bar{q}}\left[F\left(q_{i}+\bar{p}-p_{i}\right)\right]^{n-1} f\left(q_{i}\right) d q_{i}
\end{aligned}
$$

\footnotetext{
${ }^{4}$ Notice that the buyer's utility might be negative even with her best buy: we are implicitly ruling out the outside option of not purchasing the good at all.
} 
Notice that, defining $V(x)=\operatorname{Pr}\left\{\max _{j \neq i}\left\{q_{j}\right\}-q_{i} \leq x\right\}$ and $v(x)=V^{\prime}(x)$ (i.e. the density of the difference between the highest quality of $i$ 's competitors and $i$ 's own quality, which can be written explicitly using convolutions) the maximization problem can also be written as

$$
\max _{p_{i}} p_{i} V\left(\bar{p}-p_{i}\right)
$$

Optimising with respect to $p_{i}$ the first order condition $\frac{\partial H\left(p_{i}, \bar{p}\right)}{\partial p_{i}}=0$ can be expressed as

$$
V\left(\bar{p}-p_{i}\right)-p_{i} v\left(\bar{p}-p_{i}\right)=0
$$

Imposing $p_{i}=\bar{p}=p^{*}$ we obtain the (candidate) Nash equilibrium of this game:

$$
p^{*}=\frac{V(0)}{v(0)}=\frac{1 / n}{(n-1) \int_{\underline{q}}^{\bar{q}}\left[F\left(q_{i}\right)\right]^{n-2} f^{2}\left(q_{i}\right) d q_{i}}
$$

Obviously, the second order condition $\frac{\partial^{2} H\left(p^{*}, p^{*}\right)}{\partial p_{i}^{2}}<0$ or, $2 v(0)-\frac{V(0)}{v(0)} v^{\prime}(0)>0$ needs to hold for the above equilibrium price to be the solution of problem (2): the density $v(\cdot)$ has to be differentiable in zero for this to make sense, otherwise we need the function appearing in (3) to be decreasing around $p^{*}$. A condition which bypasses the possible non-smoothness of $v(\cdot)$ and which ensures that $p^{*}$ is a Nash equilibrium, is that the distribution $V(\cdot)$ be log-concave.$^{5}$ In turn, a condition which ensures this is that $f(x)$ be log-concave $\AA^{6}$ (see An [1] and Bagnoli and Bergstrom [2]). Interestingly, it also turns out to be sufficient for stability, as we shall explain in section 3.

Formula (4) emphasises the way in which the optimal bidding strategy is affected by the (common) beliefs of suppliers over the buyer's preferences, represented by the

\footnotetext{
${ }^{5}$ The log-concavity of $V$ means that the ratio $\frac{v}{V}$ is decreasing. This implies that, if the first order condition holds, i.e.

$$
V\left(\bar{p}-p_{i}\right)\left(1-p_{i} \frac{v\left(\bar{p}-p_{i}\right)}{V\left(\bar{p}-p_{i}\right)}\right)=0
$$

then, for a positive quantity $\delta$

$$
1-\left(p_{i}+\delta\right) \frac{v\left(\bar{p}-p_{i}-\delta\right)}{V\left(\bar{p}-p_{i}-\delta\right)}<0
$$

and

$$
1-\left(p_{i}-\delta\right) \frac{v\left(\bar{p}-p_{i}+\delta\right)}{V\left(\bar{p}-p_{i}+\delta\right)}>0
$$

which guarantees that the first order condition selects indeed a maximum. Notice that the above means that the objective function in (2) is pseudo-concave (so its critical point is a global maximum).

${ }^{6}$ Indeed log-concavity of the density $f_{X}$ implies the log-concavity of the density of the mirror image $f_{-X}$ and of the distribution function $F$ (see Bagnoli and Bergstrom [2, Theorem 8 and Theorem 1 respectively). In turn it is easy to see that the distribution function $F^{n}$ and its associated density $n F^{n-1} f$ are both log-concave. The convolution of $n F^{n-1} f$ and $f_{-X}$ gives the density $v$ which is again log-concave (see An [1, Corollary 1). Finally, Theorem 1 of Bagnoli and Bergstrom [2] implies that $V$ is also $\log$-concave.
} 
distribution function $F$. This solution is the same as the equilibrium price of the model of monopolistic competition proposed by Perloff and Salop [12] (see equations 12 and 13, p. 110). They analyse the outcome of competition in a differentiated market with $n$ firms and $L$ consumers. Each consumer is identified by specific tastes, represented by an $n$-dimensional vector of values, one for each firm, and corresponding to independent draws from the same probability function $F$. Consumers maximize their net utility, given by the difference between each firm's value and the correspondent price. So the oligopolist's maximization problem in this product differentiated market is formally equivalent to the bidder's maximization problem in a non-binding auction. The only exception is that oligopolists face $L$ consumers, while bidders compete to serve a unique buyer. However this difference does not affect the price equilibrium that is identical for these two games.

Perloff and Salop [12] prove that there can be at most one symmetric price equilibrium. However, in the case of $n>2$ the possibility of equilibria in asymmetric prices is not ruled out, even though costs are identical for each firm. Conversely, in the case in which $n=2$ the existence of a multiplicity of equilibria is not admissible, so the symmetric equilibrium is surely unique. The case with only two firms is particularly interesting because it has a formal correspondence with the model of price competition in the classic duopoly à la Hotelling. In the next section we will show how the distribution representing buyers' preferences is very close to the distribution of consumers along the Hotelling line.

\subsection{Optimal price strategy in an Hotelling game with non uni- form consumers}

Imagine there are two suppliers, $\mathrm{A}$ and $\mathrm{B}$, having production costs equal to 0 and located at either end of a Hotelling line of unit length. Consumers are characterized on the basis of their location parameter $\theta \in[0,1]$, and they are distributed on this Hotelling line according to a cumulative distribution $G(\theta)$, having a strictly positive density $g(\theta)$ over the interior of the support. Their utility function when they buy the product from supplier $i$ is equal to:

$$
U=v-p_{i}-b d_{i} \quad i=A, B
$$

where $v$ is their reservation price for each good, $p_{i}$ is the price charged by supplier $i$, $d_{i}$ is the distance from supplier $i$, where $d_{A}=\theta, d_{B}=1-\theta, b$ is the linear cost of transport. In such a setting, given a price pair $\left(p_{A}, p_{B}\right)$, we can define $\widetilde{\theta}$ as the consumer indifferent between supplier A and B, where:

$$
\widetilde{\theta}=\frac{b+p_{B}-p_{A}}{2 b}
$$


As a consequence all the consumers on the left of $\widetilde{\theta}$ prefer supplier A, while those on the right prefer supplier B. Therefore, the maximization problems of both suppliers are:

$$
\max _{p_{A} \leq v-b} p_{A}[G(\widetilde{\theta})] \quad \text { and } \quad \max _{p_{B} \leq v-b} p_{B}[1-G(\widetilde{\theta})]
$$

If we assume that consumers' reservation price, $v$, is sufficiently high, so that in equilibrium all of them buy some product, we have that in the Nash equilibrium of this game suppliers' optimal price strategies are:

$$
p_{A}=2 b \frac{G\left(\theta^{*}\right)}{g\left(\theta^{*}\right)} \quad \text { and } \quad p_{B}=2 b \frac{1-G\left(\theta^{*}\right)}{g\left(\theta^{*}\right)}
$$

where $\theta^{*}$ must satisfy the following implicit equation

$$
\theta^{*}=\frac{1}{2}+\frac{1-G\left(\theta^{*}\right)}{g\left(\theta^{*}\right)}
$$

Remark that this solution is also valid if we assume that suppliers A and B compete in a market with a single buyer, whose position is unknown to them. If $G(\theta)$ represents their common beliefs over his possible position the maximization problems are unchanged, except for the fact that in this case suppliers maximize their expected profit. On the basis of this new interpretation of the Hotelling game we can state the following proposition:

Proposition 1 The Nash-equilibrium of the auction game with concealment of buyer's private information when there are only two suppliers is equivalent to the Nash-equilibrium of the Hotelling game if

i) buyer's position $\theta$ is a function of suppliers' quality in the auction game, $\theta=\frac{b+q_{B}-q_{A}}{2 b}$ and

ii) suppliers' beliefs over $\theta$ are consistent with their beliefs over the initial qualities.

Proof. In order to prove the result we need to derive $g(\theta)$. First we define $z=$ $q_{B}-q_{A}$. $z$ is then distributed as the difference between suppliers' qualities and its density has positive values on the support $[-b,+b]$ according to the convolution

$$
h(z)=\int_{-\infty}^{+\infty} f(z+q) f(q) d q
$$

where we are assuming $f(\cdot)$ to be identically zero outside the support $[\underline{q}, \bar{q}]$. Now we can note that $\theta$ is a monotone transformation of the random variable $z$. In fact:

$$
\theta=\frac{1}{2}+\frac{z}{2 b}
$$


As a consequence, $\theta$ is distributed over the support $[0,1]$ according to the following density function:

$$
g(\theta)=2 b h(2 b \theta-b)
$$

It is easy to note that $h$, and consequently $g$, are symmetric functions. This fact implies that $G(1 / 2)=1 / 2$ and consequently condition (6) is satisfied for $\theta=1 / 2$ Substituting this value in (5) we obtain:

$$
p_{A}=p_{B}=\frac{2 b G\left(\frac{1}{2}\right)}{g\left(\frac{1}{2}\right)}=\frac{1}{2 h(0)}=\frac{1}{2 \int_{\underline{q}}^{\bar{q}} f^{2}(q) d q}
$$

But this solution is coincident with formula (4) when $n=2$.

Therefore the conclusion of Perloff and Salop [12] and Gal-Or et al [7], according to which firms' optimal price strategy depends on the distribution of buyers' tastes, corresponds to the result achieved by Kim [10], that the optimal price strategy of duopolists in an Hotelling setup depends on the distribution of consumers' location along Main Street.

\section{Economic dynamics under bounded rationality.}

In this section we shall embed the above analysis into a simple dynamic framework, meant to be a rough indicator of whether the Nash equilibrium derived above is bound to be actually reached if agents are either boundedly rational and/or are unsure about the other players' rationality. Each seller is supposed to be participating in a sequence of auctions in which, at each time, the seller's quality parameter is drawn from the same distribution. In the stage game each seller has a best strategy which depends on the bids of his competitors. The reaction function of a generic seller can be derived by solving for $p_{i}$ in the first order condition applied to the objective function in (1):

$$
\int_{\underline{q}}^{\bar{q}}\left[\prod_{j \neq i} F\left(q_{i}+p_{j}-p_{i}\right)\right] f\left(q_{i}\right) d q_{i}-p_{i} \int_{\underline{q}}^{\bar{q}} \sum_{j \neq i} f\left(q_{i}+p_{j}-p_{i}\right) \prod_{h \neq j, i} F\left(q_{i}+p_{h}-p_{i}\right) f\left(q_{i}\right) d q_{i}=0
$$

Notice that the best response of seller $i, p_{i}^{*}$, does not depend on the competitors' bids which have no chance of winning. More precisely $p_{h}-\min _{j}\left\{p_{J}\right\}>\bar{q}-q$ implies that $\frac{\partial p_{i}^{*}}{\partial p_{h}}=0$. While the converse is not always true we can observe that, generically, a change in the strategy of a possibly winning competitor affects the optimal bid $p_{i}^{*}$. So when price dispersion is sufficiently low, a bidder's best response is influenced by the full vector of competitors' bids.

Therefore in this context each bidder's optimal strategy is not focused on beating the best price, as in standard first price auction. However, even with a restricted number 
of competitors it is quite difficult to calculate explicitly the best response function (see equation (7)). For this reason we hypothesise that agents simplify their problem by treating their opponents as if they were all bidding a price equal to a mean of the full vector of competitors' prices and we let sellers update their expectations about its value adaptively. Results are robust to having subject-specific weighted averages; due to the heavier required notation we shall stick to simple means in the following (see Footnote 7).

Summing up, sellers at each time $t$, solve the same optimization problem using the first order conditions in (3) given a different value for the opponents' mean price, and will therefore be using the same reaction function evaluated at these different values. The symmetry imposed over the sellers implies that each shall have the same reaction function, $R()$. So seller $i$ at time $t$ will choose $p_{t, i}=R\left(\bar{p}_{t,-i}^{e}\right)$ and compute the expected mean price of the opponents according to

$$
\begin{aligned}
\bar{p}_{t,-i}^{e} & =\bar{p}_{t-1,-i}^{e}+\alpha_{i}\left(\bar{p}_{t-1,-i}-\bar{p}_{t-1,-i}^{e}\right) \\
\bar{p}_{t,-i} & =\sum_{j \neq i} \frac{p_{t, j}}{n-1} \\
p_{t, i} & =R\left(\bar{p}_{t,-i}^{e}\right)
\end{aligned}
$$

Notice that we allow some behavioral heterogeneity in that $\alpha_{i}$ may vary across different sellers. These equations define the following $n$-dimensional discrete dynamical system in $\bar{p}_{t,-i}^{e}$

$$
\left\{\begin{array}{c}
\bar{p}_{t+1,-1}^{e}=\bar{p}_{t,-1}^{e}+\alpha_{1}\left(\frac{\sum_{j \neq 1} R\left(\bar{p}_{t,-j}^{e}\right)}{n-1}-\bar{p}_{t,-1}^{e}\right) \\
\ldots \\
\bar{p}_{t+1,-n}^{e}=\bar{p}_{t,-n}^{e}+\alpha_{n}\left(\frac{\sum_{j \neq n} R\left(\bar{p}_{t,-j}^{e}\right)}{n-1}-\bar{p}_{t,-n}^{e}\right)
\end{array}\right.
$$

which possesses a single steady state whereby

$$
\bar{p}_{t,-i}^{e}=p^{*} \quad i=1, \ldots, n
$$

where $p^{*}$ is the Nash equilibrium derived above in (4). Stability of the steady state can be characterised as usual studying the Jacobian matrix of the system evaluated at the steady state, provided the reaction function is differentiable at $p^{*}$ : in this case we have

$$
J_{n}=\left[\begin{array}{cccc}
1-\alpha_{1} & \alpha_{1} \frac{R^{\prime}\left(p^{*}\right)}{n-1} & \cdots & \alpha_{1} \frac{R^{\prime}\left(p^{*}\right)}{n-1} \\
\ldots & \cdots & \ddots & \cdots \\
\alpha_{n} \frac{R^{\prime}\left(p^{*}\right)}{n-1} & \alpha_{n} \frac{R^{\prime}\left(p^{*}\right)}{n-1} & \cdots & 1-\alpha_{n}
\end{array}\right]
$$

We aim at giving conditions on the underlying parameters of the model that ensure that the spectral radius of $J_{n}$ is less than one for any choice of the vector of gain parameters 
$\alpha_{1}, \ldots, \alpha_{n}$ in $(0,1)^{n}$ or, symmetrically, provide conditions under which a suitable choice of such parameters implies instability of the steady state and therefore that the Nash equilibrium will not be reached as $t$ grows. In particular it is well known that if $\|\cdot\|$ is a matrix norm on $J_{n}$ and $\rho\left(J_{n}\right)$ is its spectral radius then

$$
\rho\left(J_{n}\right) \leq\left\|J_{n}\right\|
$$

Consider for example $\|\underset{n \times n}{A}\|=\max _{i} \sum_{j=1}^{n}\left|a_{i j}\right|$. In the case of the above matrix $J_{n}$ it is

$$
\left\|J_{n}\right\|=\max _{i} 1-\alpha_{i}+\alpha_{i}\left|R^{\prime}\left(p^{*}\right)\right|
$$

Therefore $\left|R^{\prime}\left(p^{*}\right)\right| \leq 1$ implies $\rho\left(J_{n}\right) \leq 1$ for all possible choices of $\alpha_{i}$ and therefore stability of the steady state7. So it is interesting to establish conditions ensuring a bound on the (absolute value of the) derivative of the reaction function in $p^{*}$. This is what we do in Proposition 2. But first, we need to set conditions granting differentiability of $R$ on the steady state.

Lemma 1 For the problem (2) differentiability of the reaction function at $p^{*}$ holds if and only if

$$
\begin{array}{lll}
f(\bar{q})=f(\underline{q})=0 & \text { when } & n=2 \\
f(\bar{q})=0 & \text { when } & n>2
\end{array}
$$

Proof. We now want to ascertain the differentiability of $v$ i.e.

$$
v(z)=\left\{\begin{array}{ccc}
0 & \text { if } & z<\underline{q}-\bar{q} \\
\int_{\underline{q}-z}^{\bar{q}}(n-1)[F(q+z)]^{n-2} f(q+z) f(q) d q & \text { if } & \underline{q}-\bar{q} \leq z<0 \\
\int_{\underline{q}-z}^{q}(n-1)[F(q+z)]^{n-2} f(q+z) f(q) d q & \text { if } & 0 \leq z \leq \bar{q}-\underline{q} \\
0 & \text { if } & z>\bar{q}-\underline{q}
\end{array}\right.
$$

Let $n=2$. Using Leibnitz's rule we get

$$
\begin{aligned}
& v^{\prime}\left(0^{-}\right)=f^{2}(\underline{q})+\int_{\underline{q}}^{\bar{q}} f^{\prime}(q) f(q) d q=\frac{f^{2}(\bar{q})}{2}+\frac{f^{2}(\underline{q})}{2} \\
& v^{\prime}\left(0^{+}\right)=-f^{2}(\bar{q})+\int_{\underline{q}}^{\bar{q}} f^{\prime}(q) f(q) d q=-\left(\frac{f^{2}(\bar{q})}{2}+\frac{f^{2}(\underline{q})}{2}\right)
\end{aligned}
$$

therefore $v^{\prime}(0)$ exist if and only if $f^{2}(\underline{q})=f^{2}(\bar{q})=0$, in which case $v^{\prime}(0)=0$.

\footnotetext{
${ }^{7}$ Note that this would not change if in 10 heterogeneous weighted averages replaced the arithmetic means. Indeed such generalisation would not alter the matrix norm 11 and therefore the stability conditions.
} 
Instead, when $n>2$

$$
\begin{aligned}
& v^{\prime}\left(0^{-}\right)=\int_{\underline{q}}^{\bar{q}}(n-1)(n-2)[F(q)]^{n-3} f^{3}(q)+(n-1)[F(q)]^{n-2} f^{\prime}(q) f(q) d q \\
& v^{\prime}\left(0^{+}\right)=-(n-1) f^{2}(\bar{q})+v^{\prime}\left(0^{-}\right)
\end{aligned}
$$

so $v^{\prime}(0)$ exist if and only if $f^{2}(\bar{q})=0$ and $v^{\prime}\left(0^{-}\right) \neq \pm \infty$.

Proposition 2 Consider the problem (2) under condition (12). When $n=2$ we have $R^{\prime}\left(p^{*}\right)=\frac{1}{2}$; in this case the dynamical system $(10)$ is always locally stable. When $n>2$ condition $R^{\prime}\left(p^{*}\right)<1$ always holds, while $R^{\prime}\left(p^{*}\right)>-1$ (and therefore local stability) holds if and only if

$$
\frac{v^{\prime}(0)}{v(0)^{2}}<\frac{3}{2} n
$$

Proof. Under differentiability of the density $v$ we can apply implicit differentiation to (3) to get:

$$
R^{\prime}\left(p^{*}\right)=\frac{v(0)-p^{*} v^{\prime}(0)}{2 v(0)-p^{*} v^{\prime}(0)}
$$

Therefore, when $n=2$, Lemma 1 shows that $v^{\prime}(0)=0$, so 14 implies $R^{\prime}\left(p^{*}\right)=\frac{1}{2}$ as stated.

When $n>2$ we have

$$
R^{\prime}\left(p^{*}\right)<1 \Leftrightarrow \frac{v(0)-p^{*} v^{\prime}(0)}{2 v(0)-p^{*} v^{\prime}(0)}<1
$$

which always holds, given the second order condition $2 v(0)-p^{*} v^{\prime}(0)>0$ and the fact that $v(0)=\int_{\underline{q}}^{\bar{q}}(n-1)[F(q)]^{n-2} f^{2}(q) d q>0$.

Finally:

$$
\begin{aligned}
R^{\prime}\left(p^{*}\right)>-1 & \Leftrightarrow \frac{v(0)-p^{*} v^{\prime}(0)}{2 v(0)-p^{*} v^{\prime}(0)}>-1 \\
& \Leftrightarrow \frac{v^{\prime}(0)}{v^{2}(0)}<\frac{3}{2} n
\end{aligned}
$$

The above result, implying that the $n=2$ case is a threshold above which stability is not necessarily granted, is reminiscent of classic results from the literature on dynamics in the Cournot model such as Theocharis [16]. Remark that the conditions for stability when $n>2$ can be violated only if the reaction function at $p^{*}$ is decreasing. Notice that $R^{\prime}\left(p^{*}\right)=-\frac{\partial^{2} H\left(p^{*}, p^{*}\right)}{\partial \bar{p} \partial p_{i}} / \frac{\partial^{2} H\left(p^{*}, p^{*}\right)}{\partial p_{i}^{2}}$ can be negative only if $\frac{\partial^{2} H\left(p^{*}, p^{*}\right)}{\partial \bar{p} \partial p_{i}}$ is negative, given the second order condition $\frac{\partial^{2} H\left(p^{*}, p^{*}\right)}{\partial p_{i}^{2}}<0$. This means that only under strategic 
substitutability at equilibrium can the system fail to converge to the Nash equilibrium. In other words it has to be the case that a more aggressive strategy by suppliers $j \neq i$ (i.e. a lower bid) raises $i$ 's marginal profit. Vice versa strategic complementarity at equilibrium is always associated with a (dynamically) stable Nash equilibrium.

A specific example in which (13) fails is as follows: let $n=3$ and consider the beta density function with parameters $a=3 / 4, b=3$

$$
f(x)=\frac{x^{-\frac{1}{4}}(1-x)^{2}}{\int_{0}^{1} x^{-\frac{1}{4}}(1-x)^{2} d x}
$$

We get $\frac{v^{\prime}(0)}{v^{2}(0)} \simeq 5.4604$, thus violating condition $\sqrt{13}$, and $p^{*}=\frac{V(0)}{v(0)} \simeq \frac{1 / 3}{1.5595}=0.21374$ implying

$$
R^{\prime}\left(p^{*}\right)=\frac{v(0)-p^{*} v^{\prime}(0)}{2 v(0)-p^{*} v^{\prime}(0)} \simeq-4.5591
$$

In this case $8^{8}$ the system's Jacobian at the steady state is

$$
J_{3}=\left[\begin{array}{ccc}
1-\alpha_{1} & \alpha_{1} \frac{R^{\prime}\left(p^{*}\right)}{2} & \alpha_{1} \frac{R^{\prime}\left(p^{*}\right)}{2} \\
\alpha_{2} \frac{R^{\prime}\left(p^{*}\right)}{R^{\prime}} & 1-\alpha_{2} & \alpha_{2} \frac{R^{\prime}\left(p^{*}\right)}{2} \\
\alpha_{3} \frac{R^{\prime}\left(p^{*}\right)}{2} & \alpha_{3} \frac{R^{\prime}\left(p^{*}\right)}{2} & 1-\alpha_{3}
\end{array}\right]
$$

which for the above specific value of $R^{\prime}\left(p^{*}\right)$ has eigenvalues outside the unit circle for suitable values 9 of $\alpha_{1}, \alpha_{2}, \alpha_{3}$.

Our last point regards dynamics in the $n=2$ case when the density $v(\cdot)$ is not differentiable in zero. In this case, which happens if either $f(\bar{q})$ or $f(q)$ are non-zero, $v^{\prime}\left(0^{-}\right)>0$ and $v^{\prime}\left(0^{+}\right)<0$ so in turn $R^{\prime}\left(p^{*-}\right)<0<R^{\prime}\left(p^{*+}\right)<1$. This implies that, locally, a perturbation (either positive or negative) from $p^{*}$ will eventually lead the dynamics to a decreasing path towards $p^{*}$ which therefore turns out to be locally stable.

\section{Conclusions.}

We have shown that the non-binding auction model analysed by Gal-or et al. [7] is formally equivalent to the differentiated market studied by Perloff and Salop [12] and,

\footnotetext{
${ }^{8}$ Notice that a case like this would not be possible if $f$ were log-concave. Indeed that would make $V$ log-concave as well and therefore, given $p^{*}=\frac{V(0)}{v(0)}$,

$$
v^{2}(0)-V(0) v^{\prime}(0)>0 \Rightarrow v(0)-p^{*} v^{\prime}(0)>0
$$

and $R^{\prime}\left(p^{*}\right)>0$ as a consequence. This argument also shows that, under log-concavity the reaction function has positive slope and, because such slope cannot exceed 1, local stability is ensured.

${ }^{9}$ For example $\alpha_{1}=0.3, \alpha_{2}=0.4, \alpha_{3}=0.5$ imply the following eigenvalues: $1.5777,1.4285$, -1.2062 .
} 
for the $n=2$ case, to the generalisation of the Hotelling duopoly recently proposed by Kim [10]. We have examined more in depth the symmetric equilibrium of this class of games. In particular, we have emphasised how a sufficient condition for both the existence and the stability of such equilibrium requires the log-concavity of the probability density of bidders' (oligopolists') qualities. However, there exist distribution functions for which stability can be violated. This is the result of an extension of these models to a dynamic framework in which bidders behave according to some expectation over the prices of their competitors (summarized by their mean), and update these expectations adaptively on the basis of the data from previous auctions. The steady state of such a system is unstable only if, in equilibrium, bidders' reaction functions are negatively sloped, i.e. under strategic substitutability. The dynamic analysis of oligopoly thus far has been focused mainly on the Cournot model. This work then represent an attempt to adopt the same approach in analysing other forms of market imperfections. Moreover, we have shown how this methodology can be applied also in an auction framework, at least when bidders have no private information and the Nash equilibrium is in pure strategies. Future research can be devoted to investigate the potentiality of such analysis in these kind of settings.

\section{References}

[1] AN, M.Y. "Logconcavity versus Logconvexity: A Complete Characterization". Journal of Economic Theory, Vol. 50 (1998), pp. 350-369.

[2] BAGnoli, M. and BerGSTROM, T. "Log-Concave Probability and its Applications." Economic Theory, Vol. 26 (2005), pp. 445-469.

[3] BARKLEY ROSSER JR., J. "The Development Of Complex Oligopoly Dynamics Theory." in Oligopoly Dynamics: Models and Tools, edited by Tönu Puu and Irina Sushko, Springer, 2002.

[4] BOARD, S. "Revealing Information in Auctions: the Allocation Effect." Economic Theory, Vol. 38 (2009), pp. 125-135.

[5] CASOn, T., GANGADhARAN, L. and DUkE, C. "A Laboratory Study of Auctions for Reducing Non-Point Source Pollution." Journal of Environmental Economics and Management, Vol. 46 (2003), pp. 446-471.

[6] CHAN, C., LAPlaGNE, P. and APPELS, D. "The Role of Auctions in Allocating Public Resources." Productivity Commission Staff Research Paper, Productivity Commission, Melbourne, 2003.

[7] GAL-OR, E., GAL-OR, M., and DUKES, A. "Optimal Information Revelation in Procurement Schemes." RAND Journal of Economics, Vol. 38 (2007), pp. 400-418. 
[8] GANUZA, J.J. "Ignorance Promotes Competition: an Auction Model with Endogenous Private Valuations." RAND Journal of Economics, Vol. 35 (2004), pp. 583-598.

[9] KATOK, E. and WAMBACH, A. "Collusion in Dynamic Buyer-Determined Reverse Auctions." mimeo, 2008.

[10] KIM, J. "The Intensity of Competition in the Hotelling Model: a New Generalisation and Applications." mimeo, 2007.

[11] MILGROM, P. and WEBER, R. "A Theory of Auctions and Competitive Bidding." Econometrica, Vol. 50 (1982), pp. 1089-1122.

[12] PERLOFF, J. and SALOP, S. "Equilibrium with Product Differentiation", Review of Economic Studies, 52, (1985), 107-120

[13] PUU, T. "The Chaotic Duopolists Revisited." Journal of Economic Behavior and Organization, Vol. 33 (1998), 385-394.

[14] PUU, T., GARDINI, L. "Hotelling type duopoly and oligopoly" in Oligopoly Dynamics: Models and Tools, edited by Tönu Puu and Irina Sushko, Springer, 2002.

[15] REZENDE, L. "Biased Procurement Auctions." Economic Theory, Vol. 38 (2009), pp. 169-185.

[16] THEOCHARIS, R. "On the Stability of the Cournot Solution on the Oligopoly Problem." Review of Economic Studies, Vol. 27 (1960), pp. 133-134. 